\title{
DETERMINATION OF INITIAL EIGENORBITS FOR ASTEROIDS
}

\author{
KARRI MUINONEN \\ Observatory, University of Helsinki, Helsinki, Finland \\ ANDREA MILANI \\ Department of Mathematics, University of Pisa, Pisa, Italy \\ AND \\ EDWARD BOWELL \\ Lowell Observatory, Flagstaff, Arizona, USA
}

\begin{abstract}
We consider initial determination of asteroid orbits in the case of small numbers of observations and short observational arcs. For asteroids having long arcs, we can assess the orbital uncertainties with the help of the covariance matrix that, in the phase space of the orbital elements, defines a set of probability ellipsoids. In initial orbit determination, because of the nonlinear characteristics and multiple solutions of the inversion problem, we cannot readily use the covariance matrix to estimate the orbital uncertainties. However, by computing the eigenvalues and eigenvectors of the formal correlation matrix (that is, by deriving the eigenorbits), we can discern the most indeterminate orbital elements. Here we solve the eigenproblem for 17 single-apparition asteroids.
\end{abstract}

\section{Introduction}

We consider initial determination of asteroid orbits, and solve for the eigenvalues and eigenvectors of the formal correlation matrix. Similar methods have earlier been applied to comet P/Hale-Bopp and asteroid (719) Albert (Muinonen, 1996; Bailey et al., 1996).

We make use of two different methods for orbit determination before differential correction: one is the Gaussian method for three observations (e.g., Dubyago, 1961), whereas the other is a systematic search through the topocentric distances of two observations. Occasionally, to obtain a first

I. M. Wytrzyszczak, J. H. Lieske and R. A. Feldman (eds.),

Dynamics and Astrometry of Natural and Artificial Celestial Bodies, 191, 1997.

(C) 1997 Kluwer Academic Publishers. Printed in the Netherlands. 
orbit, we need to add random noise to the observations. We improve all or a subset of orbital elements by means of differential correction (e.g., Muinonen and Bowell, 1993). Accordingly, the correction is either complete or incomplete. Our present orbit determination software derives from Muinonen and Bowell (1993), Milani et al. (1995), and Carpino and Knežević (1996).

Dubyago (1961) gave a concise history of orbit determination - one of the oldest inversion problems in astronomy. The problem involves six nonlinear parameters, rendering it a challenging inversion even for modern computational methods. As pointed out by, for example, Marsden (1991), initial orbit determination from three observations can be quite indeterminate: there are often multiple solutions, among which it is impossible to choose the right one.

Väisälä (1939) provided methods for orbit determination from, for example, two or three observations. In the latter case, Väisälä iterated toward an orbit solution by making hypotheses of the topocentric distance and comparing the $z$-components of a normalized velocity vector. In the former case, he assumed that the asteroid is at perihelion or aphelion. Bowell et al. (1989) and Jedicke (1996) have considered orbit determination from one position and one motion vector, and obtained promising results close to opposition.

In Section 2, we outline the computation of eigenorbits and, in Section 3, derive initial orbits for 17 short-arc single-apparition asteroids: 1077 T-1, 2738 P-L, 2872 P-L, 2838 P-L, 1986 PS $_{1}$, 1987 AN, $1990 \mathrm{HD}_{6}$, $1990 \mathrm{HL}_{6}, 1990 \mathrm{RB}_{18}, 1990 \mathrm{RM}_{18}, 1990 \mathrm{RV}_{17}, 1992 \mathrm{DW}_{10}, 1992 \mathrm{EY}_{4}$, $1992 \mathrm{LO}, 1993 \mathrm{PT}_{6}, 1995 \mathrm{BU}_{11}$, and $1995 \mathrm{DM}_{6}$. In Section 4 , we analyze the eigenvalues and eigenvectors of their formal correlation matrices, and draw conclusions in Section 5.

\section{Definition of Eigenorbits}

We denote the orbital elements at a given epoch by $\mathbf{P}=\left(a, e, i, \Omega, \omega, M_{0}\right)^{T}$ ( $T$ is transpose). The elements are, respectively, the semimajor axis, eccentricity, inclination, longitude of ascending node, argument of perihelion, and the mean anomaly. The three angular elements $i, \Omega$, and $\omega$ are currently referred to the ecliptic at equinox J2000.0. The determination of the final orbital elements by means of complete differential correction yields the covariance matrix $\Sigma$ (Muinonen and Bowell, 1993). In the case of incomplete differential correction, we obtain $\Sigma$ by running, at the end, complete correction without introducing changes in the elements.

In the phase space of the orbital elements, the real, symmetric, and positive definite covariance matrix $\Sigma$ defines a set of ellipsoids centered at 
the least-squares orbit $\mathbf{P}_{\mathbf{l}}$,

$$
\Delta \chi^{2}(\mathbf{P})=\Delta \mathbf{P}^{T} \Sigma^{-1} \Delta \mathbf{P}=\Delta \chi_{0}^{2}, \quad \Delta \mathbf{P}=\mathbf{P}-\mathbf{P}_{\mathrm{ls}},
$$

where $\Delta \chi_{0}^{2}$ is a constant. We express the differences $\Delta \mathbf{P}$ in terms of the standard deviations $\sigma_{j}=\sqrt{\Sigma_{j j}}(j=1, \ldots, 6)$ and use the dimensionless correlation matrix $C$; with the help of the diagonal standard deviation matrix $S$,

$$
\begin{aligned}
\Delta \mathbf{Q} & =S^{-1} \Delta \mathbf{P}, \quad S_{j k}=\sigma_{j} \delta_{j k}, \\
C & =S^{-1} \Sigma S^{-1}, \quad j, k=1, \ldots, 6,
\end{aligned}
$$

where $\delta_{j k}$ is the Kronecker symbol. We thus define the ellipsoids by

$$
\Delta \mathbf{Q}^{T} C^{-1} \Delta \mathbf{Q}=\Delta \chi_{0}^{2}
$$

in which all the parameters are dimensionless.

Implicit in making use of the correlation matrix is the idea that, probabilistically, the orbital elements contribute uniformly to the eigenproblem. The eigenvalues $\lambda_{1}, \ldots, \lambda_{6}$ for the correlation matrix are normalized variances along the principal axes of the ellipsoid, the directions of the axes being given by the orthonormal eigenvectors $\mathbf{X}_{1}, \ldots, \mathbf{X}_{6}$,

$$
C \mathbf{X}_{j}=\lambda_{j} \mathbf{X}_{j}, \quad j=1, \ldots, 6 .
$$

An eigenorbit is one that lies on a principal axis of the ellipsoid.

In what follows, the orbital uncertainties cannot be readily inferred from the covariance matrices computed here in the linear approximation. Instead of covariances, correlations, and standard deviations, we thus discuss pseudo-covariances, pseudo-correlations, and pseudo-deviations.

\section{Orbit Determination}

In Table 1 , we show the numbers of observations and nights, the observational arcs, the R.A. and Dec. residuals, and sample sets of orbital elements. All observations were obtained from the Minor Planet Center in May 1996. In orbit determination, we gave preference to convergent rather than realistic solutions. For four asteroids, the differentially corrected orbital elements reproduced the observed positions with practically zero residuals.

Gaussian orbit determination yielded solutions for the eleven asteroids 1077 T-1, 2738 P-L, 2782 P-L, 2838 P-L, 1987 AN, $1990 \mathrm{HD}_{6}, 1990 \mathrm{HL}_{6}$, $1992 \mathrm{DW}_{10}, 1992 \mathrm{EY}_{4}, 1992 \mathrm{LO}$, and $1995 \mathrm{DM}_{6}$. From these, complete differential correction converged for all but $1077 \mathrm{~T}-1$ and $1995 \mathrm{DM}_{6}$. For 
TABLE 1. We summarize the number of observations $N$, number of nights $N_{\mathrm{n}}$, observational arc $T$ (d), standard deviations of the R.A. and Dec. residuals $\sigma_{\alpha}$ and $\sigma_{\delta}$ (arcsec), and the orbital elements (semimajor axis in $\mathrm{AU}$, angular elements in degrees). The epochs are the following (TDT): $1077 \mathrm{~T}-1,1971-03-26.0 ; 2738 \mathrm{P}-\mathrm{L}$, 2782 P-L, and 2838 P-L, 1960-09-26.0; 1986 PS $_{1}$, 1986-08-03.0; 1987 AN, 1987-01-03.0; $1990 \mathrm{HD}_{6}$ and $1990 \mathrm{HL}_{6}, 1990-05-01.0 ; 1990 \mathrm{RB}_{18}, 1990 \mathrm{RM}_{18}$, and $1990 \mathrm{RV}_{17}$, 1990-09-10.0; $1992 \mathrm{DW}_{10}$ and $1992 \mathrm{EY}_{4}, 1992-03-03.0 ; 1992 \mathrm{LO}, 1992-06-05.0$; $1993 \mathrm{PT}_{6}, 1993-08-16.0 ; 1995 \mathrm{BU}_{11}, 1995-02-02.0 ; 1995 \mathrm{DM}_{6}, 1995-02-27.0$.

\begin{tabular}{|c|c|c|c|c|c|c|c|c|}
\hline Name & $N, N_{\mathrm{n}}, T$ & $\sigma_{\alpha}, \sigma_{\delta}$ & $a$ & $e$ & $i$ & $\Omega$ & $\omega$ & $M_{0}$ \\
\hline 1077 T-1 & $5,3,3.0$ & $0.46,0.62$ & 2.83 & .256 & 24.24 & 353.60 & 37.04 & 138.42 \\
\hline 2738 P-L & $6,3,3.0$ & $0.25,0.25$ & 3.13 & .182 & 10.66 & 185.23 & 137.87 & 33.51 \\
\hline 2782 P-L & $7,4,5.0$ & $0.32,0.33$ & 3.03 & .058 & 3.88 & 118.30 & 75.67 & 176.95 \\
\hline 2838 P-L & $6,3,4.0$ & $0.48,0.43$ & 3.53 & .690 & 4.64 & 20.79 & 124.62 & 304.72 \\
\hline $1986 \mathrm{PS}_{1}$ & $6,3,3.1$ & $0.68,0.74$ & 2.45 & .377 & 4.99 & 350.87 & 111.37 & 254.74 \\
\hline $1987 \mathrm{AN}$ & $5,5,5.1$ & $0.90,0.86$ & 2.45 & .426 & 1.10 & 161.71 & 246.00 & 24.65 \\
\hline $1990 \mathrm{HD}_{6}$ & $3,3,3.0$ & 0,0 & 2.67 & .424 & 5.47 & 91.44 & 301.20 & 276.84 \\
\hline $1990 \mathrm{HL}_{6}$ & $3,3,3.0$ & 0,0 & 2.08 & .631 & 3.39 & 94.06 & 312.88 & 279.40 \\
\hline $1990 \mathrm{RB}_{18}$ & $3,3,2.0$ & $0.37,0.72$ & 3.96 & .318 & 14.56 & 292.90 & 271.75 & 55.26 \\
\hline $1990 \mathrm{RM}_{18}$ & $3,3,2.0$ & $1.53,0.62$ & 2.47 & .190 & 1.68 & 159.17 & 235.14 & 292.65 \\
\hline $1990 \mathrm{RV}_{17}$ & $3,3,2.0$ & $1.72,1.62$ & 2.80 & .079 & 5.43 & 297.49 & 101.15 & 269.94 \\
\hline $1992 \mathrm{DW}_{10}$ & $3,3,4.9$ & 0,0 & 2.32 & .286 & 3.57 & 314.12 & 284.97 & 311.07 \\
\hline $1992 \mathrm{EY}_{4}$ & $3,3,5.0$ & 0,0 & 3.47 & .530 & 23.01 & 149.52 & 228.21 & 80.06 \\
\hline $1992 \mathrm{LO}$ & $5,3,3.1$ & $0.45,0.49$ & 2.44 & .301 & 7.21 & 208.99 & 319.03 & 45.69 \\
\hline $1993 \mathrm{PT}_{6}$ & $10,4,4.0$ & $2.12,2.25$ & 2.70 & .335 & 4.71 & 316.37 & 315.15 & 32.16 \\
\hline $1995 \mathrm{BU}_{11}$ & $8,3,9.0$ & $0.38,0.26$ & 2.41 & .246 & 3.08 & 346.97 & 240.68 & 304.72 \\
\hline $1995 \mathrm{DM}_{6}$ & $12,5,6.0$ & $0.29,0.45$ & 3.15 & .048 & 23.78 & 349.21 & 325.36 & 215.21 \\
\hline
\end{tabular}

$1077 \mathrm{~T}-1$, fixing the argument of perihelion and carrying out incomplete differential correction took us close to a more probable orbit solution that was also convergent in complete differential correction. For $1995 \mathrm{DM}_{6}$, fixing the eccentricity, incomplete differential correction converged well. For 2738 P-L, 2782 P-L, 2838 P-L, $1990 \mathrm{HD}_{6}, 1990 \mathrm{HL}_{6}, 1992 \mathrm{DW}_{10}, 1992 \mathrm{LO}$, and $1995 \mathrm{DM}_{6}$, systematic searches yielded orbit solutions nonlinearly interrelated to those adopted here.

It was difficult to obtain orbits for $1986 \mathrm{PS}_{1}, 1990 \mathrm{RB}_{18}, 1990 \mathrm{RM}_{18}$, $1990 \mathrm{RV}_{17}, 1993 \mathrm{PT}_{6}$, and $1995 \mathrm{BU}_{11}$ via Gaussian orbit determination, whereas systematic searches produced good first orbits for all these asteroids. For $1990 \mathrm{RB}_{18}, 1990 \mathrm{RM}_{18}$, and $1990 \mathrm{RV}_{17}$, we had to add uniformly distributed random noise to both R.A. and Dec. and repeat the systematic search numerous times before finding realistic orbit solutions. In these three cases, we did not obtain fully satisfactory fits: the residuals show systematic shifts and high rms values. 
TABLE 2. Examples of orbit solutions for 2738 P-L (I), 2782 P-L (II), and $2838 \mathrm{P}-\mathrm{L}$ (III) with maximum sky-plane residuals less than $10^{\prime \prime}$. We give the semimajor axis in $\mathrm{AU}$, the inclination in degrees, and note the persistent high-eccentricity solutions.

\begin{tabular}{rcrcccrcr} 
& I & \multicolumn{1}{c}{ II } & \multicolumn{1}{c}{ III } \\
\hline \multirow{2}{*}{$a$} & $e$ & \multicolumn{1}{c}{$i$} & $a$ & $e$ & \multicolumn{1}{c}{$i$} & $a$ & $e$ & \multicolumn{1}{c}{$i$} \\
\hline 2.817 & 0.213 & 6.751 & 3.376 & 0.361 & 3.609 & 2.740 & 0.098 & 1.900 \\
3.053 & 0.139 & 10.760 & 3.074 & 0.155 & 3.713 & 2.701 & 0.070 & 2.010 \\
7.349 & 0.799 & 11.864 & 3.067 & 0.027 & 3.827 & 2.716 & 0.266 & 2.673 \\
3.955 & 0.646 & 48.737 & 3.240 & 0.223 & 4.020 & 10.297 & 0.951 & 9.031 \\
7.838 & 0.834 & 64.258 & 4.122 & 0.490 & 4.327 & 77.335 & 0.997 & 16.652 \\
\hline
\end{tabular}

Generally, the elements were far from determinate: to illustrate the complicated structure of the a posteriori probability density of the orbital elements, Table 2 gives examples of multiple solutions for the asteroids $2738 \mathrm{P}$ $\mathrm{L}, 2782 \mathrm{P}-\mathrm{L}$, and $2838 \mathrm{P}-\mathrm{L}$ in the order of increasing inclination.

\section{Pseudo-Deviations, Eigenvalues, and Eigenvectors}

When computing the pseudo-covariances, we assumed R.A. and Dec. residual standard deviations of precisely $11^{\prime \prime} 0$ for all 17 sample asteroids, independently of the a posteriori standard deviations in Table 1 . We made use of biased estimators, replacing the denominator $2 N-6$ in Muinonen and Bowell (1993) by $2 N$ ( $N$ is the number of observations).

We divided the sample asteroids into three broad categories based on the first eigenvector of the pseudo-correlation matrix. In the first category, the first eigenvector projects practically equally onto the normalized orbital element axes. In the second category, the eigenvector projects slightly less onto one or more of the element axes and, without exception, the second eigenvector projects maximally onto the same axes. In the third category, the first eigenvector is almost perpendicular to one or more of the axes.

There are seven members in the first category: $2838 \mathrm{P}-\mathrm{L}, 1990 \mathrm{HL}_{6}$, $1990 \mathrm{RM}_{18}, 1990 \mathrm{RV}_{17}, 1992 \mathrm{EY}_{4}, 1992 \mathrm{LO}$, and $1995 \mathrm{BU}_{11}$. The corresponding eigenvalues are the largest in the entire set of sample asteroids, approaching the maximum possible value of 6.0 . Furthermore, the smallest eigenvalues and eigenvectors relate to orbital elements that show the strongest mutual pseudo-correlations, whereas the second eigenvalue and eigenvector relate to elements that show the weakest (but usually still strong) pseudo-correlations with the other elements. 
TABLE 3. The pseudo-deviations $\tilde{\sigma}_{1}, \ldots, \tilde{\sigma}_{6}$ of $a(\mathrm{AU}), e, i\left({ }^{\circ}\right), \Omega\left({ }^{\circ}\right), \omega\left({ }^{\circ}\right)$, and $M_{0}\left({ }^{\circ}\right)$, and the sorted eigenvalues $\lambda_{1}, \ldots, \lambda_{6}$ (e.g., 3.54(-4) means $3.54 \cdot 10^{-4}$ ) and orthonormal eigenvectors $\mathrm{X}_{1}, \ldots, \mathrm{X}_{6}$ for the asteroids $2738 \mathrm{P}-\mathrm{L}, 2782 \mathrm{P}-\mathrm{L}$, and 2838 P-L.

\begin{tabular}{rrrrrrr} 
Name, $\left\{\tilde{\sigma}_{j}\right\}$ & $\lambda_{1}, \mathrm{X}_{1}$ & $\lambda_{2}, \mathrm{X}_{2}$ & $\lambda_{3}, \mathrm{X}_{3}$ & $\lambda_{4}, \mathrm{X}_{4}$ & $\lambda_{5}, \mathrm{X}_{5}$ & $\lambda_{6}, \mathrm{X}_{6}$ \\
\hline \multicolumn{1}{c}{$2738 \mathrm{P}-\mathrm{L}$} & 4.24 & 1.76 & $3.54(-4)$ & $1.34(-5)$ & $5.06(-8)$ & $1.56(-11)$ \\
\hline .093866 & .388998 & -.451314 & .746783 & .295124 & -.012886 & -.006894 \\
.118804 & .475851 & -.149112 & -.099017 & -.590112 & -.001755 & .627130 \\
2.270519 & -.480240 & -.109875 & .335381 & -.413729 & -.688219 & -.000012 \\
1.260186 & -.480245 & -.109825 & .333359 & -.345335 & .722701 & .067990 \\
8.722276 & -.040425 & .752042 & .392277 & .220448 & -.033226 & .478765 \\
8.459413 & -.399184 & -.429395 & -.234567 & .475020 & -.052778 & .610592 \\
\hline $2782 \mathrm{P}-\mathrm{L}$ & 5.38 & $6.23(-1)$ & $1.48(-3)$ & $1.22(-4)$ & $9.82(-8)$ & $2.30(-13)$ \\
\hline .083878 & .429256 & .122545 & -.268124 & .831950 & -.191547 & -.000628 \\
.103279 & -.430529 & .071829 & .548230 & .233001 & -.674269 & .001370 \\
.166644 & -.420563 & .280721 & .295910 & .429272 & .687375 & -.000002 \\
2.232021 & -.354114 & .722771 & -.544163 & -.142532 & -.188566 & .014822 \\
107.005366 & .406380 & .424177 & .351676 & -.153679 & .019989 & .712205 \\
118.172692 & -.403695 & -.445753 & -.346700 & .159255 & -.015156 & .701813 \\
\hline $2838 \mathrm{P}-\mathrm{L}$ & 5.98 & $1.73(-2)$ & $9.02(-6)$ & $4.70(-8)$ & $9.37(-10)$ & $2.90(-13)$ \\
\hline 3.972712 & .408136 & -.445341 & -.111254 & -.761490 & -.206611 & -.012810 \\
1.028924 & .408829 & -.054831 & -.410765 & .296271 & .101547 & -.750357 \\
9.621028 & .408681 & .211405 & .439625 & -.172799 & .751770 & .000068 \\
22.082941 & -.408692 & -.203827 & -.616921 & -.174907 & .600044 & .142084 \\
62.988257 & .407394 & .637876 & -.493131 & -.055500 & -.125264 & .406442 \\
116.301043 & .407755 & -.552758 & -.043062 & .518483 & .078088 & .501415 \\
\hline & & & & & &
\end{tabular}

There are five members in the second and third categories: 2782 P-L, $1987 \mathrm{AN}, 1990 \mathrm{HD}_{6}, 1993 \mathrm{PT}_{6}$, and $1995 \mathrm{DM}_{6}$ belong to the former, whereas $1077 \mathrm{~T}-1,2738 \mathrm{P}-\mathrm{L}, 1986 \mathrm{PS}_{1}, 1990 \mathrm{RB}_{18}$, and $1992 \mathrm{DW}_{10}$ belong to the latter category. The elements improved are most often $a, e$, and $\omega$. Again, the fifth and sixth eigenvectors relate to elements that are either strongly pseudo-correlated or whose pseudo-deviations are remarkably large.

For example, Table 3 lists the pseudo-deviations, and the sorted eigenvalues and eigenvectors for the asteroids $2738 \mathrm{P}-\mathrm{L}, 2782 \mathrm{P}-\mathrm{L}$, and $2838 \mathrm{P}-\mathrm{L}$, representing the three aforementioned categories. To find the direction of a principal axis in the phase space of the orbital elements, one needs to mul- 
tiply the eigenvector components by the pseudo-deviations. For example, the first eigenvector for $2838 \mathrm{P}-\mathrm{L}$ provides a principal axis that, among the angular elements, lies close to the $\omega-M_{0}$-plane.

The condition numbers - the ratios of the largest and smallest eigenvalues of the pseudo-correlation matrix - were high for all sample asteroids, varying from $2.72 \cdot 10^{11}$ for 2738 P-L to $2.77 \cdot 10^{15}$ for $1990 \mathrm{RV}_{17}$, and signalled the ill-posedness of the orbit-determination problem. It is, however, notable that all the eigenvalues were positive, indicating that the pseudo-correlation matrices were positive definite.

Among the angular orbital elements, the pseudo-deviations of $\omega$ and $M_{0}$ were larger than the deviations of the other angular elements. For $1990 \mathrm{RV}_{17}$, the pseudo-deviation of $M_{0}$ formally extended more than six times around a full circle. In general, the orbital elements with the largest pseudo-deviations were usually the strongest pseudo-correlators.

We computed the R.A. and Dec. residuals for formal eigenorbits of several asteroids. The residuals were large, signalling the inapplicability of the linear approximation. Thereafter, we measured the phase space distance between two orbit solutions by using the $\Delta \chi^{2}$ metric in Eq. (1): using the formal covariance matrix, the orbits were usually far from each other. For $2782 \mathrm{P}-\mathrm{L}$, though, the multiple orbit solutions are in line with the second eigenvector (Tables 1-3): the pseudo-covariances thus appear to give local information about a curve of variation in the phase space.

In conclusion, the 17 sample asteroids can be divided into four overlapping subgroups: inner-main-belt asteroids $1986 \mathrm{PS}_{1}, 1987 \mathrm{AN}, 1990 \mathrm{HL}_{6}$, $1990 \mathrm{RM}_{18}, 1992 \mathrm{DW}_{10}, 1992 \mathrm{LO}$, and $1995 \mathrm{BU}_{11}$; mid-main-belt asteroids $1077 \mathrm{~T}-1,1990 \mathrm{HD}_{6}, 1990 \mathrm{RV}_{17}$, and $1993 \mathrm{PT}_{6}$; outer-main-belt asteroids 2738 P-L, 2782 P-L, and $1995 \mathrm{DM}_{6}$; and the remaining asteroids 2838 P-L, $1990 \mathrm{RB}_{18}, 1992 \mathrm{EY}_{4}$ that have even larger semimajor axes.

\section{Conclusion}

For most of our sample asteroids, Gaussian orbit determination yielded acceptable first orbits, which we improved by means of differential correction. In many cases, the complete differential correction procedure diverged, but we were always able to find acceptable fits via incomplete differential correction, a powerful tool in initial orbit determination.

Because of the highly nonlinear nature of the initial orbit determination problem, the orbital uncertainties cannot be estimated with the help of the linear approximation. Instead, one will have to study the non-Gaussian a posteriori probability density for the orbital elements by using, e.g., Monte Carlo methods (Muinonen and Bowell, 1993). It is thus evident that, primarily as a function of the observational arc and the number of observations, there exists a boundary regime outside which the covariance analysis works 
well, but inside which the nonlinear nature of the inversion problem plays a dominant role. To find the precise validity regime of the covariance analysis is a future research goal.

We will solve the orbital covariance eigenproblem for all single-apparition asteroids, revisiting the work by Muinonen et al. (1994). However, multi-apparition asteroids play a key role in studies of initial orbit determination: by making use of subsets of observations, we can gain conclusive insight on how the initial orbital elements and eigenorbits relate to the final orbital elements.

In initial determination of asteroid orbits, a systematic search for solutions through the phase space of the orbital elements is by far the safest strategy. We will supplement that by computing, based on all known asteroids, the Bayesian a priori probability density of the orbital elements and assign a quantitative measure of reality to all orbit solutions obtained. Thus, we will replace the human reality measure, used extensively in initial orbit determination, by a rigorous mathematical measure. In the era of ever improving computational capabilities, the Bayesian approach appears attractive.

Acknowledgements. Research supported, in part, by the EU/HCM contracts CHRX-CT9 and ERB CHBG CT 940607, and NASA grant NAGW-1470.

\section{References}

Bailey, M.E., Emel'yanenko, V.V., Hahn, G., Harris, N.W., Hughes, K.A., Muinonen, K., and Scotti, J.V.: 1996, "Orbital evolution of Comet 1995 O1 Hale-Bopp", Mon. Not. R. Astron. Soc. 281, 916-924.

Bowell, E., Skiff, B.A., Wasserman, L.H., and Russell, K.S.: 1990, "Orbital information from asteroid motion vectors", in: Asteroids, Comets, Meteors III (C.-I. Lagerkvist, H. Rickman, B.A. Lindblad, M. Lindgren, eds), Uppsala Universitet, 19-24.

Carpino, M., and Knežević, Z.:, 1996, "Determination of the mass of (1) Ceres from close approaches of other asteroids", in: Dynamics, Ephemerides, and Astrometry of Solar System Bodies, IAU Symposium 172 (S. Ferraz-Mello, B. Morando, J.-E. Arlot, eds), Kluwer, Dordrecht, 203-206.

Dubyago, A.D.: 1961, The Determination of Orbits, The MacMillan Company.

Jedicke, R.: 1996, "Detection of near-Earth asteroids based upon their rates of motion", Astron. J. 111, 970-982.

Marsden, B.G.: 1991, "The computation of orbits in indeterminate and uncertain cases", Astron. J. 102, 1539-1552.

Milani, A., Carpino, M., Rossi, A., Catastini, G., and Usai, S.: 1995, "Local geodesy by satellite laser ranging: An European solution", Manuscr. Geod. 20, 123-138.

Muinonen, K.: 1996, "Orbital covariance eigenproblem for asteroids and comets", Mon. Not. R. Astron. Soc. 280, 1235-1238.

Muinonen, K., and Bowell, E.: 1993, "Asteroid orbit determination using Bayesian probabilities", Icarus 104, 255-279.

Muinonen, K., Bowell, E., and Wasserman, L.H.: 1994, "Orbital uncertainties of singleapparition asteroids", Planet. Space Sci. 42, 307-313.

Väisälä, Y.: 1939, "Eine einfache Methode der Bahnbestimmung", Mitt. Sternw. Univ. Turku, Suomalainen Tiedeakatemia, 1, 1-32. 